\title{
THE EFFECTS OF VEGETARIAN DIET ON THE CARDIOVASCULAR SYSTEM
}

\author{
Nazlıcan Cengiz ${ }^{1}$ (D), Tülay Ece Özçelik ${ }^{1}$ (D), Beyza Yılmaz ${ }^{1}$ (D) Nisanur Bayar² (D), Selma Arzu $\operatorname{Vardar}^{3}$ (D)
}

\author{
${ }^{1}$ Trakya University School of Medicine, Edirne, TURKEY \\ ${ }^{2}$ Trakya University School of Physiotherapy and Rehabilitation, Edirne, TURKEY \\ ${ }^{3}$ Department of Physiology, Trakya University School of Medicine, Edirne, TURKEY
}

\begin{abstract}
Vegetarianism is the refusal to eat meat and its products for various reasons. Furthermore, veganism is against eating and using all products derived from animals. Numerous studies have stated that plant-based nutrition reduces the risk of cardiovascular system diseases, but also cannot supply the requirement of some vitamins and minerals. Although a vegetarian diet may decrease the risk of cardiovascular diseases, the findings of the studies related to the low intake of protein, vitamins, or minerals should be taken into account in terms of harmful effects. In this review, the studies have been reviewed about the effects of plant-based nutrition on the human cardiovascular system. Keywords: Cardiovascular system, vegans, vegetarians, diet, plant proteins
\end{abstract}

\section{INTRODUCTION}

Vegetarianism is the refusal to eat meat (red meat, poultry, seafood, and the flesh of any other animal) (1). Vegetarians may be further sub-classified as vegans, pesco-vegetarians, lacto-vegetarians, lacto-ovo-vegetarians, and semi-vegetarians (2). Vegans avoid using or eating any animal products (2). Pesco-vegetarians consume fish and other seafood (2). Lacto-vegetarians eat dairy products; lacto-ovo-vegetarians eat dairy products and eggs (2). As another subgroup, semi-vegetarians usually follow a vegetarian diet but occasionally consume meat (2).

Some people think that consuming meat is unhealthy and that a vegetarian diet generally prevents diseases (3). Other common reasons for choosing vegetarian diets are animal welfare or rights, satisfying religious or spiritual needs, and saving the environment (3). The prevalence of vegetarianism varies around the world; roughly $5 \%$ of Americans, $8 \%$ of Canadians, $4.3 \%$ of Germans, and $30 \%$ of Indians follow a vegetarian diet (4-8). Vegan diet prevalence has been reported as $2 \%$ in the United States and less than $1 \%$ in Germany $(4,9)$.

Plant-based diets have documented health advantages (10). It decreases the serum cholesterol levels, blood pressure, and vegetarians have decreased cardiometabolic risk than omnivores (10). Vegetable and fruit intake frequency is inversely proportional to cardiovascular disease mortality, ischemic heart disease (IHD) mortality, stroke incidence, and stroke mortality (11). According to the studies, vegetarian diets decrease blood pressure compared to omnivorous diets (12). Lower blood pressure leads to a decrease in deaths from all causes, deaths from coronary heart disease (CHD), and stroke $(12,13)$.

In this review, the effects of deficiencies of some vitamins and minerals, effects of vegetarian diet on the cardiovascular system, possible effects of vegetarian diet on cardiac diseases, as well as the effects of soy protein, which is consumed by vegetarians, on heart function are investigated.

\section{NUTRIENT PROFILES AND NUTRITIONAL DEFICIENCY OF VEGETARIANS}

It is considered that vegetarian diets improve health and decrease the risk of cardiovascular diseases (CVDs) (14). On the other hand, a vegetarian diet may cause vitamin deficiency because vegetarians have lower intakes of protein, saturated fat, cholesterol, vitamin B12, vitamin D, zinc, calcium, and selenium (14).

Although veganism and vegetarianism exist all over the world, only in the past 50 years it was noticed that vegan and/or vegetarian diets lead to vitamin B12 deficiency (15). Vegans and vegetarians tend to have a vitamin B12 deficiency because their diets have a very low B12 content (15). Vitamin B12 can only be synthesized in nature by some bacteria found in the bowels of humans, therefore humans must provide the intake of vitamin B12 from their diet (15). Non-vegetarians receive vitamin B12 from meat, eggs, milk, and dairy products (15). Plant fertilization with manure might cause contamination with vitamin B12 synthesizing bacteria. Thus, organic vegetables can be a better source of vitamin B12 (15).

In a large-scale study, participants were stratified into non-vegetarian, semi-vegetarian, pesco-vegetarian, and strict vegetarian (16). Data on dietary patterns were collected, and nutrient profiles were presented. Results showed that pesco-vegetarians and non-vegetarians had the highest intakes of vitamin B12 and vitamin D while strict vegetarians had the lowest (16). Furthermore, intakes of calcium and iron were lowest in strict vegetarians (16). Conversely, strict vegetarians had the highest intakes of folate, soy protein, fiber, vitamin $\mathrm{C}$, and vitamin $\mathrm{E}(16)$. 
Homocysteine is an amino acid that is produced through the demethylation of methionine and animal proteins contain plenty of methionine (17). It has been revealed that plasma homocysteine concentration is one of the independent risk factors of CHD (18). Vitamin B12 and some other B vitamins are necessary for homocysteine metabolism (19). Cobalamin (B12) as cofactor and folate (B9) as co-substrate are responsible for the remethylation of homocysteine (19). The other route for homocysteine metabolism is transsulfuration and it is a process that is dependent on vitamin B6 (20). Deficiency in these vitamins may result in the increment of plasma homocysteine concentration (21). Riboflavin (B2) has a role in the metabolism of other $B$ vitamins and because of its role, riboflavin deficiency may affect homocysteine levels (22).

In a previous study, subjects were separated into groups such as vegetarians and non-vegetarians (21). Daily nutrient intakes' data was recorded and plasma homocysteine, vitamin B12, and folate levels were measured by obtaining fasting venous blood samples (21). The results of this study showed that vegetarian subjects had significantly higher mean plasma homocysteine concentration but lower vitamin B12 intakes than non-vegetarians (21). In addition, a positive correlation has been shown between animal protein intake and plasma homocysteine level in the non-vegetarian group (21).

\section{VEGETARIANISM AND CARDIOVASCULAR DISEASES}

Cardiovascular diseases refer to diseases of the heart and blood vessels (23). High intake of fat, saturated fats, and carbohydrates are important risk factors that increase the prevalence of CVDs (24). CVD is a medical term that includes diseases such as coronary heart disease (CHD), peripheral artery disease, cerebrovascular disease, rheumatic and congenital heart diseases, and venous thromboembolism CVDs are the major factor of global deaths $(25,26)$. It is responsible for one in four deaths across the world (26). The high prevalence of heart diseases was associated with lifestyle factors such as smoking, consuming refined foods, high animal fat content in the diet, and physical inactivity (27). According to a study, vegetarians have a $32 \%$ lower risk of getting CHD collated to non-vegetarians (28).

Diets containing high amounts of refined carbohydrates increase triglyceride concentration, while diets with a high fiber ratio decrease it (29). Dietary cholesterol is strongly associated with CVDs (29). The vegan diet has a cholesterol-lowering effect due to its low saturated fat content, soluble fiber, and other plant components (29). However, according to the findings of a previous study, although the total and low-density lipoprotein (LDL) cholesterol of vegans were at normal levels, high-density lipoprotein cholesterol level was low, homocysteine was high, and lipoprotein (a) level was negative in terms of coronary artery disease (CAD) (30). On the other hand, there are different opinions in studies about the effects of hyperhomocysteinemia (28-30). To understand the connection between hyperhomocysteinema and the vegan diet, the number of studies on this subject should be increased.

Atrial fibrillation has the highest prevalence in arrhythmias, and its risk factors include hypertension, CAD, and obesity (31). Plant-based nutrition reduces the likelihood of these risk factors. Improved vasodilatation, increased potassium intake, decreased blood viscosity are the main effects of plant-based diets for preventing hypertension (31). CAD is described as the blockage of the vessels that carry oxygen and nutrients to the heart (32). High-sensitivity C-reactive protein (hsCRP) is used as a marker of CAD (33). A high hsCRP level is an indicator of CAD risk (33). A study conducted with CAD patients with high hsCRP values showed that in addition to medical treatment, a vegan diet had lowered hsCRP levels (33). A vegan diet supports weight loss, glycemic control, and normalizing lipid values (33). However, studies examining medical treatment and diet together on CAD are limited (33). On the other hand, B12 deficiency is common among vegans and vegetarians (34). B12 deficiency may cause impaired carotid intima-media thickness and brachial flow-mediated endothelium-dependent dilation values compared to a similar group (34).

Vegetarian diets may not seem to be preventative for diseases, although it supports the treatment of type 2 diabetes (35). It is known that vegetarian diets combined with exercise can reduce the use of glucose in the body. Thus, plasma glucose levels and the use of glucoregulatory drugs decrease (34-36). A vegetarian diet also has beneficial effects without exercise. It reduces body weight and lowers blood lipids. Vegetarian diets are found more effective in glycemic control and treatment of type 2 diabetes compared to traditional diets $(37,38)$.

Trimethylamine $\mathrm{N}$-oxide (TMAO) has been recently thought to be a prognostic marker for developing CVDs and most of the studies focus on this metabolite (39). TMAO is the form of trimethylamine (TMA) transformed by flavin monooxygenase in the liver (39). TMA is produced from substances such as choline, L-carnitine, betaine, and trimethyl lysine in the intestine (39). TMAO is a chemical compound that can accumulate in organs such as the heart and kidney (40). TMAO and its precursors are animal food items that originate mostly from fish, meat, egg, poultry, and milk (40). The amount of TMAO component is less in vegetarians because their diet contains fewer animal foods and these precursors (41). Preclinical studies showed that TMAO directly causes damage to the heart muscle and mitochondria (40). TMAO increases the risk of thrombosis and ischemic heart failure (HF) (40). In addition, studies indicate that a vegetarian diet avoids and cures HF (42).

In a meta-analysis by Miller et al. (43), it was observed that intake of egg yolks with diet increased urine and plasma TMAO concentrations. This study showed phosphatidylcholine, which is a type of choline in eggs, transforms TMA contrary to what is believed (43). These concentrations reach the highest level with consumption of 4-6 eggs (43). But they could not demonstrate a connection with increased TMAO levels and CVDs (43).

All dairy products, eggs, red meat, and processed meat include saturated fatty acids (44). In a randomized controlled study, it was revealed that plasma LDL cholesterol levels were raised by saturated fatty acids intake (44). Recent studies showed that a low-calorie vegetarian diet decreases LDL cholesterol levels and is efficient in preventing and treating cardiovascular system diseases (45). Conversely, cheese, eggs, and yogurt were linked to significantly lower IHD risk. Poultry, milk, and fish were not related to IHD (46). In a study carried out by Key et al. (46), it has been observed that when red meat and processed meat were consumed together, heart rate and IHD risk increased (46). Consumption of eggs reduces IHD risk in men but does not affect women (46). Yogurt and cheese were invertedly linked to IHD risk, but this relation was not significant in the study (46). Fatty acids and calcium in cheese generate insoluble soaps. It is thought that when saturated fatty acid absorption decreases, calcium binds to bile acids and diminishes enterohepatic circulation and cholesterol levels (44).

Vegetarianism is also preferred in different age groups and periods. It should be taken into consideration that nutritional needs vary depending on different periods of life and physical activity. If vegetarian people do not follow a proper diet it may have unfavorable health effects. In the studies with vegan and non-vegan athletes, there is no difference found in their performances (47). It has been observed that vegan athletes do not show any deficiency 
compared to non-vegan athletes when they follow a suitable diet (47). A vegan athlete's relative wall thickness, as well as systolic and diastolic functions, are better than a non-vegan athlete's (47). Besides, a vegan athlete's end-diastolic volume and end-systolic volume are higher and stroke volume is lower than a non-vegan athlete. Measurable differences have not been seen between the muscle mass and bone density of vegan and non-vegan athletes (47). Vegan athletes have better cholesterol levels than non-vegan ones (48).

\section{SOY PROTEIN CONSUMPTION AND CARDIOVASCULAR EFFECTS}

Soy protein is a food frequently consumed by vegetarians rather than omnivores. The United States Food and Drug Administration authorized labeling of soy protein foods as reducing the risk of CVDs (49). However, it has not been definitively proven whether cholesterol efflux and macrovascular function can be changed with diets that are rich in polyphenols (50).

Soybeans contain many bioactive substances, including polyphenols and isoflavones (51). Flavonoids are grouped according to their chemical structure. These are: flavonols (quercetin, kaempferol), flavanols (catechins), flavones (apigenin), and isoflavones (daidzein, genistein) (51).

Many studies have observed that soy isoflavones reduce LDL levels, which has an important role in atherosclerosis pathogenesis $(48,52,53)$. It has been found that blood cholesterol levels increase in many animals fed with animal protein diets $(54,55)$. Contrary, it has been demonstrated that using soy protein instead of animal protein causes hypercholesterolemia (48). In the studies on isoflavones, the bioactive molecule of soy, soy isoflavones caused arterial vasodilation and lower serum cholesterol in animals, and inhibit atherosclerosis in postmenopausal monkeys $(56,57)$. Some previous studies have been conducted to separate the protein effect from the isoflavone effect $(53,58-63)$. LDL cholesterol was reduced in two of these studies. It has been shown that protein influences LDL reduction and isoflavones have no effect $(52,63)$.

Cardiac complications due to radiotherapy include subacute or chronic $\mathrm{CAD}$, congestive heart failure, ischemia, and myocardial infarction $(64,65)$. It is not enough to try to prevent interstitial fibrosis, inflammation of the myocardium, and thrombotic changes with anti-inflammatory and antithrombotic drugs (66). The inhibition of these radiation-induced damages with soy isoflavones has been proved histologically. It has been demonstrated that smooth muscle cell damage is reduced in the cardiac arteries of animals treated with radiotherapy and soy isoflavones (67). Soy isoflavones can be used as a complement to improve overall survival in patients surviving cancer with radiotherapy (67).

A prospective cohort study of Chinese women included nearly all soy foods - tofu, fried tofu, and tofu cakes. (68) The risk of cardiovascular disease, adjusted for age and energy, was reduced with soy protein intake (68). The elderly who consumed higher amounts of soy protein had a history of hypertension and a high body mass index. Women who consumed large amounts of soy protein had a lower risk of cardiovascular disease and a lower non-fatal myocardial infarction than women who consumed small amounts (68).

\section{CONCLUSION}

Studies in literature have generally shown that vegetarians have a lower risk of CVDs. Vegetarian diets contain low intakes of saturated fat and cholesterol. The consumption of food of animal origin is shown as a restrictive factor for choline, L-carnitine, betaine, trimethyl lysine levels. Therefore, the cardiovascular disease precursor TMAO is produced much less in the body. In addition, soy, which is consumed largely in vegetarian diets, is important for reducing the risk of CVDs. However, vegetarian diets can cause vitamin and mineral deficiencies since the diet does not contain animal products. Vitamin B deficiencies are especially important because vitamin $\mathrm{B}$ is responsible for homocysteine metabolism, and that plasma homocysteine concentration is one of the independent risk factors of CHD. For these reasons, it is important to regulate vegetarian diets by considering daily nutritional needs.

\section{Ethics Committee Approval: N/A}

Informed Consent: N/A

Conflict of Interest: The authors declared no conflict of interest.

Author Contributions: Concept: NC. Design: NC, TEÖ, BY, NB. Supervision: NC, TEÖ, BY, NB, SAV. Resources: NC, TEÖ, BY, NB. Materials: NC, TEÖ, BY, NB. Data collection and/or processing: NC, TEÖ, BY, NB. Analysis and/or Interpretation: NC, TEÖ, BY, NB. Literature Search: NC, TEÖ, BY, NB. Writing Manuscript: NC, TEÖ, BY, NB. Critical Review: NC, TEÖ, BY, NB.

Financial Disclosure: The authors declared that this study received no financial support.

\section{REFERENCES}

1. Ye M, Glick-Bauer M, Wechsler S. Fruit and vegetable consumption in the United States: patterns, barriers and federal nutrition assistance programs. In: Watson RR, Preedy VR, editors. Fruits, Vegetables, and Herbs. Academic Press; 2016.p.411-22.

2. White R, Frank E. Health effects and prevalence of vegetarianism. West J Med 1994;160(5):465-70

3. Lea E, Worsley A. The cognitive contexts of beliefs about the healthiness of meat. Public Health Nutr 2002;5(1):37-45.

4. Le LT, Sabaté J. Beyond meatless, the health effects of vegan diets: findings from the Adventist cohorts. Nutrients 2014;6(6):2131-47.

5. Ruby MB, Heine SJ, Kamble $\mathrm{S}$ et al. Compassion and contamination. Cultural differences in vegetarianism. Appetite 2013;71:340-8.

6. Mensink G, Barbosa CL, Brettschneider AK. Prevalence of persons following a vegetarian diet in Germany. Journal of Health Monitoring 2016;1(2):2-14.

7. Agrawal S, Millett CJ, Dhillon PK et al. Type of vegetarian diet, obesity and diabetes in adult Indian population. Nutr J 2014;13:89.

8. Shridhar K, Dhillon PK, Bowen L et al. Nutritional profile of Indian vegetarian diets - the Indian Migration Study (IMS). Nutr J 2014;13:55.

9. Pfeiler TM, Egloff B. Examining the "Veggie" personality: results from a representative German sample. Appetite 2018;120:246-55.

10. Fontana L, Meyer TE, Klein S et al. Long-term low-calorie low-protein vegan diet and endurance exercise are associated with low cardiometabolic risk. Rejuvenation Res 2007;10(2):225-34.

11. Bazzano LA, He J, Ogden LG et al. Fruit and vegetable intake and risk of cardiovascular disease in US adults: the first national health and nutrition examination survey epidemiologic follow-up study. Am J Clin Nutr 2002;76(1):93-9.

12. Yokoyama Y, Nishimura K, Barnard ND et al. Vegetarian diets and blood pressure: a meta-analysis. JAMA Intern Med 2014;174(4):577-87.

13. Whelton PK, He J, Appel LJ et al. Primary prevention of hypertension: clinical and public health advisory from the national high blood pressure education program. JAMA 2002;288(15):1882-8.

14. Larsson CL, Johansson GK. Dietary intake and nutritional status of young vegans and omnivores in Sweden. Am J Clin Nutr 2002;76(1):100-6.

15. Antony AC. Vegetarianism and vitamin B12 (cobalamin) deficiency. Am J Clin Nutr 2003;78(1):3-6.

16. Rizzo NS, Jaceldo-Siegl K, Sabate J et al. Nutrient profiles of vegetarian and nonvegetarian dietary patterns. J Acad Nutr Diet 2013;113(12):1610-9.

17. Venes D, Taber CW. Taber's Cyclopedic Medical Dictionary. In: Venes D, editor. 21st ed. Philadelphia: F.A. Davis; 2005. p.1089.

18. Boushey CJ, Beresford SA, Omenn GS et al. A quantitative assessment of plasma homocysteine as a risk factor for vascular disease. Probable benefits of increasing folic acid intakes. JAMA 1995;274(13):1049-57.

19. Gamble MV, Ahsan H, Liu X et al. Folate and cobalamin deficiencies and hyperhomocysteinemia in Bangladesh. Am J Clin Nutr 2005;81(6):1372-7.

20. Majchrzak D, Singer I, Männer M et al. B-vitamin status and concentrations of homocysteine in Austrian omnivores, vegetarians and vegans. Ann Nutr Metab 2006;50(6):485-91.

21. Huang YC, Chang SJ, Chiu YT et al. The status of plasma homocysteine and related B-vitamins in healthy young vegetarians and nonvegetarians. Eur J Nutr 2003;42(2):84-90.

22. Powers HJ. Riboflavin (vitamin B2) and health. Am J Clin Nutr 2003;77(6):135260. 
23. Ganguly P, Alam SF. Role of homocysteine in the development of cardiovascular disease. Nutr J 2015;14:6.

24. Li D, Sinclair A, Mann N et al. The association of diet and thrombotic risk factors in healthy male vegetarians and meat-eaters. Eur J Clin Nutr 1999;53(8):612-9.

25. Jack Stewart, Gavin Manmathan, Peter Wilkinson Primary. Prevention of cardiovascular disease: A review of contemporary guidance and literature. JRSM Cardiovasc Dis. 2017;1-9.

26. Lozano R, Naghavi M, Foreman K et al. Global and regional mortality from 235 causes of death for 20 age groups in 1990 and 2010: a systematic analysis for the global burden of disease study 2010. Lancet 2012;380(9859):2095-128.

27. National cholesterol education program (NCEP) expert panel on detection, evaluation, and treatment of high blood cholesterol in adults (adult treatment panel III). Third report of the national cholesterol education program (NCEP) expert panel on detection, evaluation, and treatment of high blood cholesterol in adults (adult treatment panel III) final report. Circulation 2002;106(25):3143-421.

28. Crowe FL, Appleby PN, Travis RC et al. Risk of hospitalization or death from ischemic heart disease among British vegetarians and nonvegetarians: results from the EPIC-Oxford cohort study. Am J Clin Nutr 2013;97(3):597-603.

29. Barnard ND, Cohen J, Jenkins DJA et al. A low-fat vegan diet improves glycemic control and cardiovascular risk factors in a randomized clinical trial in individuals with type 2 diabetes. Diabetes Care 2006;29(8):1777-83.

30. Waldmann A, Koschitzke JW, Leitzmann C et al. German vegan study: diet, lifestyle factors, and cardiovascular risk profile. Ann Nutr Metab 2005;49(6):366-72.

31. Storz MA, Helle P. Atrial fibrillation risk factor management with a plant-based diet: a review. J Arrhythm 2019;35(6):781-8.

32. Ganguly P, Alam SF. Role of homocysteine in the development of cardiovascular disease. Nutr J 2015;14:6.

33. Shah B, Newman JD, Woolf $\mathrm{K}$ et al. Anti-inflammatory effects of a vegan diet versus the American heart association-recommended diet in coronary artery disease trial. J Am Heart Assoc 2018;7(23):e011367.

34. Woo KS, Kwok TCY, Celermajer DS. Vegan diet, subnormal vitamin B12 status and cardiovascular health. Nutrients 2014;6(8):3259-73.

35. Anderson JW, Ward K. High-carbohydrate, high-fiber diets for insulin-treated men with diabetes mellitus. Am J Clin Nutr 1979;32(11):2312-21.

36. Barnard RJ, Jung T, Inkeles SB. Diet and exercise in the treatment of NIDDM. The need for early emphasis. Diabetes Care 1994;17(12):1469-72.

37. Kahleova $\mathrm{H}$, Matoulek $\mathrm{M}$, Malinska $\mathrm{H}$ et al. Vegetarian diet improves insulin resistance and oxidative stress markers more than conventional diet in subjects with type 2 diabetes. Diabet Med 2011;28(5):549-59.

38. Nicholson AS, Sklar M, Barnard ND et al. Toward improved management of NIDDM: a randomized, controlled, pilot intervention using a low fat, vegetarian diet. Prev Med 1999;29(2):87-91.

39. Manor O, Zubair N, Conomos MP et al. A Multi-omic Association Study of Trimethylamine N-Oxide. Cell Rep 2018;24(4):935-46.

40. Zhang Y, Wang Y, Ke B et al. TMAO: how gut microbiota contributes to heart failure. Transl Res 2021;228:109-25.

41. Koeth RA, Lam-Galvez BR, Kirsop J et al. L-Carnitine in omnivorous diets induces an atherogenic gut microbial pathway in humans. J Clin Invest 2019;129(1):373-87.

42. Hana Kahleova, Susan Levin, Neal D Barnard. Vegetarian dietary patterns and cardiovascular disease. Prog Cardiovasc Dis 2018;61(1):54-61.

43. Miller CA, Corbin KD, da Costa KA et al. Effect of egg ingestion on trimethylamine-N-oxide production in humans: a randomized, controlled, dose-response study. Am J Clin Nutr 2014;100(3):778-86.

44. Bergeron N, Chiu S, Williams PT et al. Effects of red meat, white meat, and nonmeat protein sources on atherogenic lipoprotein measures in the context of low compared with high saturated fat intake: a randomized controlled trial. Am J Clin Nutr 2019;110(1):24-33

45. Francesco Sofi, Monica Dinu, Giuditta Pagliai et al. Low-calorie vegetarian versus Mediterranean diets for reducing body weight and improving cardiovascular risk profile: CARDIVEG study (cardiovascular prevention with vegetarian diet). Circulation 2018;137(11):1103-13.
46. Key TJ, Appleby PN, Bradbury KE et al. Consumption of meat, fish, dairy products, and eggs and risk of ischemic heart disease. Circulation 2019;139(25):283545.

47. Król W, Price S, Śliż D et al. A vegan athlete's heart - is it different? Morphology and function in echocardiography. Diagnostics 2020;10(7):477.

48. Leischik R, Spelsberg N. Vegan triple-ironman (raw vegetables/fruits). Case Rep Cardiol 2014;2014(7):317246.

49. Beavers KM, Jonnalagadda SS, Messina MJ. Soy consumption, adhesion molecules, and pro-inflammatory cytokines: a brief review of the literature. Nutr Rev 2009;67(4):213-21.

50. Richter CK, Skulas-Ray AC, Fleming JA et al. Effects of isoflavone-containing soya protein on ex vivo cholesterol efflux, vascular function and blood markers of CVD risk in adults with moderately elevated blood pressure: a dose-response randomised controlled trial. Br J Nutr 2017;117(10):1403-13.

51. Ramdath DD, Padhi EMT, Sarfaraz S et al. Beyond the cholesterol-lowering effect of soy protein: a review of the effects of dietary soy and its constituents on risk factors for cardiovascular disease. Nutrients. 2017;9(4):324

52. Massimiliano Ruscica, Chiara Pavanello, Sara Gandini et al. Effect of soy on metabolic syndrome and cardiovascular risk factors: a randomized controlled trial. Eur J Nutr. 2018;57(2):499-511.

53. Jenkins DJA, Kendall CWC, Jackson CJC et al. Effects of high- and low-isoflavone soyfoods on blood lipids, oxidized LDL, homocysteine, and blood pressure in hyperlipidemic men and women. Am J Clin Nutr 2002;76(2):365-72.

54. Kritchevsky D. Vegetable protein and atherosclerosis. J Am Oil Chem Soc 1979;56(3):135-40.

55. Carroll KK. Hypercholesterolemia and atherosclerosis: effects of dietary protein. Fed Proc 1982;41(11):2792-6.

56. Anthony MS, Clarkson TB, Williams JK. Effects of soy isoflavones on atherosclerosis: potential mechanisms. Am J Clin Nutr 1998;68:1390-3.

57. Clarkson TB, Anthony MS, Morgan TM. Inhibition of postmenopausal atherosclerosis progression: a comparison of the effects of conjugated equine estrogens and soy phytoestrogens. J Clin Endocrinol Metab 2001;86(1):41-7.

58. Crouse JR, Morgan T, Terry JG et al. A randomized trial comparing the effect of casein with that of soy protein containing varying amounts of isoflavones on plasma concentrations of lipids and lipoproteins. Arch Intern Med 1999;159(17):2070-6.

59. Dent SB, Peterson CT, Brace LD et al. Soy protein intake by perimenopausal women does not affect circulating lipids and lipoproteins or coagulation and fibrinolytic factors. J Nutr 2001;131(9):2280-7.

60. Meinertz H, Nilausen K, Hilden J. Alcohol-extracted, but not intact, dietary soy protein lowers lipoprotein(a) markedly. Arterioscler Thromb Vasc Biol 2002;22(2):312-6

61. Steinberg FM, Guthrie NL, Villablanca AC et al. Soy protein with isoflavones has favorable effects on endothelial function that are independent of lipid and antioxidant effects in healthy postmenopausal women. Am J Clin Nutr 2003;78(1):12330 .

62. Gardner CD, Newell KA, Cherin R et al. The effect of soy protein with or without isoflavones relative to milk protein on plasma lipids in hypercholesterolemic postmenopausal women. Am J Clin Nutr 2001;73(4):728-35.

63. Lichtenstein $\mathrm{AH}$, Jalbert $\mathrm{SM}$, Adlercreutz $\mathrm{H}$ et al. Lipoprotein response to diets high in soy or animal protein with and without isoflavones in moderately hypercholesterolemic subjects. Arterioscler Thromb Vasc Biol 2002;22(11):1852-8.

64. Gagliardi G, Constine LS, Moiseenko V et al. Radiation dose-volume effects in the heart. Int J Radiat Oncol Biol Phys 2010;76:77-85.

65. Darby SC, Ewertz M, McGale P et al. Risk of ischemic heart disease in women after radiotherapy for breast cancer. N Engl J Med 2013;368(11):987-98.

66. Stewart FA, Seemann I, Hoving S et al. Understanding radiation-induced cardiovascular damage and strategies for intervention. Clin Oncol 2013;25(10):617-24.

67. Dominello MM, Fountain MD, Rothstein SE et al. Radiation injury to cardiac arteries and myocardium is reduced by soy isoflavones. J Radiat Oncol 2017;6(3):307-15.

68. Zhang X, Shu XO, Gao YT et al. Soy food consumption is associated with lower risk of coronary heart disease in Chinese women. J Nutr 2003;133(9):2874-8. 